\title{
FutureJournal
}

\section{enários Prospectivos das Universidades Corporativas no Brasil - 2030}

\author{
1Eduardo Spada \\ ${ }^{2}$ Sergio Henrique Arruda Cavalcante Forte
}

\section{RESUMO}

Em um ambiente altamente competitivo, o desenvolvimento de habilidades pessoais críticas aos negócios é fator crucial para a perenidade das organizações. Para isto, ao longo dos anos, as instituições empresariais públicas e privadas brasileiras têm procurado estimular e investir na formação profissional e nas habilidades dos funcionários e parceiros institucionais. Este trabalho contempla a elaboração de cenários prospectivos para as universidades corporativas no Brasil para os próximos 13 anos (2017-2030), visando subsidiar as organizações brasileiras para enfrentarem qualquer uma dessas possibilidades da melhor forma possível ou, até mesmo, criar condições para que se modifiquem suas probabilidades de ocorrência no sentido favorável às organizações a que elas pertencem. A natureza desta pesquisa configura-se como qualitativa e descritiva com a aplicação de um questionário a um grupo de quatro pesquisadores em Educação Corporativa e 15 executivos de UCs, de acordo com o modelo de prospecção de cenários prospectivos, tendo sido identificadas 16 variáveis, das quais 15 pertencem ao cenário otimista e/ou realista e apenas uma ao cenário pessimista, possibilitando às Universidades Corporativas compor suas estratégias em médio e longo prazos, reduzindo suas vulnerabilidades.

Palavras-chave: Educação corporativa. Universidade corporativa. Cenários prospectivos.

\footnotetext{
${ }^{1}$ Mestre em Administração de Empresas pela Universidade de Fortaleza - UNIFOR, Ceará, (Brasil). E-mail: eduardo.spada@cimentoapodi.com.br Orcid id: https://orcid.org/0000-00015601-9382

2 Doutor em Administração de Empresas pela Fundação Getúlio Vargas - FGV, São Paulo, (Brasil). E-mail: sergioforte@unifor.br Orcid id: https://orcid.org/0000-0002-8406-0910
} 


\title{
Prospective Scenarios of Corporate Universities in Brazil - 2030
}

\begin{abstract}
In a highly competitive environment, personal skills development for businesses is a key factor to ensure the continuous success of the organizations. For this reason, Brazilian public and private institutions have sought to stimulate and invest in vocational education, employee skills, and institutional partnerships. This study involves the development of prospective scenarios for corporate universities in Brazil over the next 13 years (2017-2030), to subsidize the Brazilian organizations to deal with any of such scenarios in the best way possible, or even to create favorable conditions for them. A qualitative and descriptive research was carried out by using a survey submitted to a group of four researchers and 15 expert in Corporate Education or executives from UCs, according to the proposed model, having been identified 16 variables for the construction of prospective scenarios for Brazilian Corporate Universities, in which 15 of them belongs to the optimistic/realistic scenarios and only one of them to the pessimistic one, making it possible to compose their strategies in medium and long terms, reducing vulnerabilities for Corporate Universities.
\end{abstract}

Keywords: Corporate education. Corporate university. Prospective scenarios 


\section{INTRODUÇÃO}

A forma como as organizações estão buscando na capacitação e no desenvolvimento contínuo de seus colaboradores, uma fonte de vantagem competitiva sustentável tem chamado a atenção de parte da literatura na área de administração. Elementos como informação, conhecimento e aprendizado têm se tornado cada vez mais relevantes nos processos organizacionais e no desempenho estratégico das organizações (EBOLI, 2008). Nesse contexto, a Universidade Corporativa (UC) vem sendo institucionalizada como uma estratégia empresarial capaz de promover vantagem competitiva, contribuindo com a transformação de oportunidades em negócios por meio do conhecimento desenvolvido compartilhado continuamente entre as pessoas que fazem parte ou estão em contato com as organizações: funcionários, clientes, fornecedores, parceiros, comunidade, sociedade, entre outros (EBOLI, 2004; MEISTER, 1999).

Allen e Mcgee (2004) definem a UC como uma organização educacional que faz parte de outra organização, cujo principal objetivo é algo além da educação e para Eboli (2012), a universidade corporativa é o elemento representativo da educação corporativa nas organizações, ou seja, a universidade corporativa consolida, em sua estrutura, o processo da educação corporativa.

De acordo com Meister (1999), foi a partir da segunda metade do século $X X$ que empresas norte-americanas (pequenas e grandes) começaram a dedicar-se com maior profundidade aos processos de aprendizagem visando aperfeiçoar as rotinas de trabalho. Foi nas empresas de tecnologia, com grandes investimentos em Pesquisa e Desenvolvimento - P\&D e necessidades constantes de treinamento, que a educação corporativa apresentou seus programas mais consistentes.

Segundo Eboli (2004), o sistema de educação corporativa (EC) viabiliza uma atuação profissional diferenciada, estimulando o colaborador a construir ou modificar a realidade organizacional por meio da reflexão crítica. Portanto, a EC representa uma escolha estratégica das organizações. Especialistas apontam que, para cumprir o seu papel, a EC deve apresentar referências educacionais sólidas, apoiadas em metodologias eficientes e alinhadas aos objetivos estratégicos do negócio. Ou seja, os objetivos da educação 
corporativa devem ser considerados estratégicos, pois contribuem para atingir vantagem competitiva empresarial e os objetivos globais de desempenho estratégico.

Em relação às medidas ou indicadores de desempenho estratégico, há um consenso entre diversos autores da área de administração estratégica, sobre a necessidade de ampliação de medidas tradicionais, em geral baseadas na rentabilidade da empresa. Sob esse ponto de vista, são necessários múltiplos indicadores de desempenho, capazes de avaliar, de forma abrangente e multidimensional, os efeitos ou resultados gerados pelas organizações (MATITZ; BULGACOV, 2011). Portanto, ainda de acordo com Matitz e Bulgacov (2011), considerando a UC como uma escolha estratégica, os resultados provenientes de sua implantação devem ser incluídos na análise do desempenho multidimensional da empresa e podem contribuir para uma compreensão ampliada do conceito e da operacionalização das medidas de desempenho estratégico. Assim, ao compreender os resultados esperados com sua implantação, possibilita-se auxiliar no esclarecimento da forma como o desempenho estratégico tem sido conceituado e operacionalizado na prática organizacional, além de indicar possibilidades teóricas de ampliação da natureza do construto.

Nesse contexto de aumento da importância da EC e, consequentemente, do aumento da quantidade de UCs e do escopo de atuação, voltado às questões de aprendizado organizacional, salienta-se a importância do estudo de cenários prospectivos para identificar possíveis possibilidades do ambiente aos quais as UCs estão inseridas, possibilitando a elaboração de estratégias preventivas para minimizar os riscos decorrentes de cenários desfavoráveis.

Diante disto e tendo em vista a relevância das UCs como vantagem competitiva e para o cumprimento dos objetivos estratégicos, assim como a necessidade de compreender o seu papel, a pesquisa empreendida busca responder a seguinte pergunta: Quais os possíveis cenários futuros para as Universidades Corporativas no Brasil no horizonte 2030? Esta pesquisa tem como objetivo geral identificar os cenários prospectados para o horizonte 2030 para as Universidades Corporativas no Brasil.

Buscando desdobramentos ao objetivo geral, definiu-se como objetivo específico identificar os eventos que mais impactam na elaboração dos cenários para as UCs brasileiras. 
Neste sentido, a pesquisa contribui teoricamente e empiricamente para a área, na medida em que há uma ausência de estudos que construam cenários prospectivos paras as UCs. Grande parte das pesquisas identificadas em UCs centram seus estudos no panorama atual sem se preocupar em prever ou prospectar futuros destas, bem como não se encontrou estudos de cenários no objeto de estudo Universidades Corporativas. Além disso, a pesquisa apresenta-se relevante, devido à atualidade do tema, em que se observa que a EC por meio das UCs se apresenta como agente de mudanças do comportamento organizacional e auxilia no desenvolvimento individual e institucional, alicerçadas no conhecimento. Assim, espera-se que este trabalho apoie os gestores estratégicos de Recursos Humanos e de Universidades Corporativas em suas ações e façam as Universidades formais refletirem sobre o avanço das UCs nas organizações.

\section{UNIVERSIDADE CORPORATIVA (UC)}

A General Motors Institute e a General Electric foram as duas primeiras empresas nos Estados Unidos a criarem Universidades Corporativas, respectivamente em 1940 e 1950 (MORIN; RENAUD, 2004)

A Universidade Corporativa surge no Brasil em 1992 com a implantação da Academia ACCOR, como um sistema de apoio à gestão de pessoas por competência, sendo seguida por outras organizações, como Brahma (EBOLI, 2008).

Os resultados positivos gerados pelo processo da educação continuada estimularam outras empresas a investirem na implantação de universidades corporativas e, em dados de 2012, há cerca de 600 organizações brasileiras com uma estrutura de Universidade Corporativa efetivamente implantada (EBOLI, 2012).

A UC é um dos elementos que irá auxiliar as empresas a migrar para a Sociedade do Conhecimento. Neste sentido, Rodriguez (2003) diz que ela está orientada para o desenvolvimento de competências críticas para que as estratégias da organização sejam alcançadas, estando voltada diretamente para o resultado das empresas.

Neste caso, o investimento na capacitação das pessoas é visto como uma das formas de abrir novas oportunidades de mercado e gerar novos 
negócios, proporcionando assim um impacto direto nos produtos ou serviços oferecidos.

Alperstedt (2001) explica que, na expressão "universidade corporativa", o uso do termo "corporativo" significa que a universidade é vinculada a uma corporação e que serviços educacionais não constituem seu principal objetivo. Já o termo "universidade" não deve ser entendido dentro do contexto do sistema de ensino superior, formal, no qual designa a educação de estudantes e o desenvolvimento de pesquisa em várias áreas do conhecimento, uma vez que "universidade corporativa" oferece instrução específica, sempre relacionada à área de negócio da própria organização. Portanto, para Alperstedt (2001), o termo "universidade" para essas empresas seria basicamente um artifício de marketing.

Várias universidades corporativas por efeito mimético têm adotado algumas das tipificações de universidades tradicionais, por meio de reitores, catálogo de cursos, escolas dentro da universidade e até alguns campi (ALLEN; MCGEE, 2004).

Para Meister (1999), há cinco forças que explicam o avanço das universidades corporativas: 1 .

O advento e a consolidação da economia do conhecimento; 2. A emergência da organização não-hierárquica, enxuta e flexível; 3. A redução do prazo de validade do conhecimento; 4. O novo foco na capacidade de empregabilidade ocupacional para a vida toda em lugar do emprego para a vida toda; e 5. Uma mudança fundamental no mercado da educação global. Tais tendências compõem o cenário que torna compreensível as sete competências básicas nas organizações, definidas como "a soma de qualificações, conhecimento e conhecimento implícito, necessária para superar o desempenho da concorrência" e fundamental para a empregabilidade do indivíduo.

A seguir, apresenta-se o Quadro 1 com pesquisas e fontes recentes contemplando temas sobre Universidade Corporativa:

Quadro 1 - Pesquisas e fontes em Universidade Corporativa por tema 


\begin{tabular}{|l|l|}
\hline Tema & Fonte \\
\hline $\begin{array}{l}\text { Atuação e alinhamento } \\
\text { Estratégico }\end{array}$ & $\begin{array}{l}\text { Castro; Eboli (2013); Freitas-Dias; Albuquerque } \\
\text { (2014); Globalccu (2017). }\end{array}$ \\
\hline $\begin{array}{l}\text { Competição com } \\
\text { Universidades Tradicionais }\end{array}$ & Globalccu (2017). \\
\hline Alianças e Parcerias & Globalccu (2017). \\
\hline Internacionalização de UCs & Castro; Eboli (2013). \\
\hline Lançamentos de novas UCs & Globalccu (2017). \\
\hline $\begin{array}{l}\text { Público-alvo (Value Chain } \\
\text { Education) }\end{array}$ & $\begin{array}{l}\text { Barbosa (2016); Castro; Eboli (2013); Freitas-Dias; } \\
\text { Albuquerque (2014); Guerci, Bartezzaghi, Solari } \\
\text { (2010); Marinelli (2013); Parker (2017). }\end{array}$ \\
\hline $\begin{array}{l}\text { Desenvolvimento de } \\
\text { Lideranças }\end{array}$ & $\begin{array}{l}\text { Barbosa (2016); Castro; Eboli (2013); Globalccu } \\
\text { (2017); Marinelli (2013) }\end{array}$ \\
\hline $\begin{array}{l}\text { Metodologias de } \\
\text { Educação/EAD }\end{array}$ & Barbosa (2016); Delloite (2016). \\
\hline $\begin{array}{l}\text { Certificação formais de } \\
\text { diplomas }\end{array}$ & Globalccu (2017). \\
\hline Nível Organizacional & Castro; Eboli (2013). \\
\hline $\begin{array}{l}\text { Tecnologia mobile e digital } \\
\text { e Inovação }\end{array}$ & $\begin{array}{l}\text { Abel; Li (2012); Castro; Eboli (2013); Globalccu } \\
\text { (2017). }\end{array}$ \\
\hline Orçamento & Castro; Eboli (2013) \\
\hline Efetividade da EC & $\begin{array}{l}\text { Freitas-Dias; Albuquerque (2014); Allen; Mcgee } \\
\text { (2004); Barbosa (2016); Freitas-Dias; Carvalho } \\
\text { (2014); Globalccu (2017); Kirkpatrick (2010). }\end{array}$ \\
\hline $\begin{array}{l}\text { Retorno sobre o } \\
\text { Investimento }\end{array}$ & Allen; Mcgee (2004); Marinelli (2013). \\
\hline $\begin{array}{l}\text { Mensuração de Impactos } \\
\text { Sociais e Ambientais }\end{array}$ & $\begin{array}{l}\text { Castro; Eboli (2013); Marinelli (2013); Globalccu } \\
\text { (2017). }\end{array}$ \\
\hline Fonte:
\end{tabular}

Fonte: Elaborado pelos Autores da pesquisa (2017).

Assim, ainda de acordo com Paton, Taylor e Storey (2004), identificar cenários prospectivos para as UCs visa possibilitar a elaboração de estratégias preventivas para minimizar riscos de cenários desfavoráveis.

\section{CENÁRIOS}

Antes de iniciar a discussão sobre os cenários prospectivos, faz-se necessário apresentar algumas definições e objetivos, um breve histórico da evolução dos estudos prospectivos, as características e tipologias dos cenários, os cenários prospectivos no Brasil, e, por fim, o processo de criação 
dos cenários, embora não seja objetivo desta pesquisa fornecer uma visão completa do método de cenários.

\subsection{Definições, Objetivos, Vantagens e Tipologias de Cenários}

Apesar das nuanças e diferenças de interpretação, existe um certo consenso sobre as definições e os objetivos de cenários.

O termo cenário deriva do termo teatral grego "cenários, que quer dizer o roteiro de uma peça de teatro. Os elementos principais para a conceituação e o entendimento da técnica são os mesmos do teatro, quais sejam: cenários, cenas, trajetórias e atores (MORITZ, 2004).

Uma das definições mais abrangentes sobre cenários encontrada na literatura contemporânea, é a de Godet (1993), para quem a técnica de cenários é um conjunto formado pela descrição coerente de uma situação futura e pelo encaminhamento imaginado e criado dos acontecimentos que permitem passar da situação de origem à situação futura.

Os cenários não são previsões, pois não é possível prever o futuro com um razoável grau de certeza. Ao contrário da previsão tradicional de pesquisa de mercado, os cenários apresentam alternativas do futuro (FREITAS FILHO et al., 2013; SCHWARTZ, 2000). Portanto, não é uma realidade futura, mas pode ser um meio para representá-lo (DURANCE; GODET, 2010).

Dentre as vantagens no uso de cenários se destacam o aumento da velocidade, a flexibilidade em responder às mudanças e o estímulo ao pensamento conjunto e sistemático dos gestores. Já as principais barreiras, segundo Schoemaker (1993), referem-se à dificuldade de fazer com que as pessoas abandonem a descrença em alternativa de futuro, os custos de tempo, recursos humanos e materiais, a falta de comprometimento da liderança e as falhas de comunicação entre os desenvolvedores de cenários e os gestores.

Quanto à tipologia, os cenários podem ser qualificados por sua natureza ou probabilidade e classificados como normativos ou exploratórios (GODET, 2000; MARCIAL; GRUMBACH, 2002), sendo necessário distinguir uma fase exploratória de identificação dos desafios do futuro e uma fase normativa de definição das escolhas estratégicas possíveis e desejáveis (GODET, 2000). 


\subsection{A Evolução do Estudo de Cenários}

Ao longo dos últimos anos, possibilidades alternativas sobre as técnicas de cenários prospectivos foram ofertadas pelas obras e contribuições de Berger (1964), Godet (1993), Heijden (2004), Kahn (1969), Schwartz (2000) e Wack (1985).

A prospectiva diz respeito a um termo francês utilizado por Berger nos anos 50, representando uma forma de se concentrar sobre o futuro, imaginando-o insólito e absurdo, mas extraindo deduções sobre o presente.

Segundo Heijden (2004), a prospecção de cenários transformou-se e atingiu novas dimensões no início dos anos 1970, com o trabalho do cenarista francês Pierre Wack, na área de planejamento do futuro da Royal Dutch Shell, salientando a necessidade de criar uma nova ferramenta estratégica que auxiliasse a empresa no seu planejamento em longo prazo frente à crise do petróleo na década de 70 do século passado.

No início da década de 80 , estudos de cenários entraram no campo do planejamento estratégico, tanto no meio público como no meio privado. A metodologia ficou conhecida devido a alguns trabalhos realizados pelas consultorias Battelle (Emilio Fontela), Sema (Michel Godet) e SRI (Ian Wilson e Tom Mandel), passando a ser utilizada por uma grande quantidade de empresas ao redor do mundo (GODET, 2000; ROUBELAUT, 2000).

Alguns autores também contribuíram com o estudo de cenários, direcionando para vantagem competitiva como Michael Porter; Peter Senge em organização de aprendizagem; e Henry Mintzberg, em planejamento estratégico (COATES; DURANCE; GODET, 2010).

As mudanças ocorridas a partir do século $\mathrm{XX}$ tornaram cada vez mais difíceis a sobrevivência das organizações no ambiente competitivo (OLIVEIRA; FORTE, 2011). Além disso, percebe-se nos últimos anos a nova economia de mercado considerando fortemente o avanço tecnológico, a revolução verde e a maior esperança pela a vida, sendo fatores ambientais, desenvolvimento sustentável e o bem-estar da população temas de grande repercussão, mas previstos por poucos futuristas (GODET, 2010).

Em 1988, com o surgimento da Global Business Network (GBN) empresa de prospectiva criada por Peter Schwartz e Pierre Wack - inicia-se a popularização do emprego dos cenários como instrumento de gestão 
estratégica para as empresas anglo saxônicas e sua divulgação e expansão em todo o mundo organizacional a partir da década de 90.

Evidencia-se, assim, que o estudo de cenários tem se mostrado cada vez mais relevante para a formulação estratégica das organizações, uma vez que a análise tradicional baseada na observância do passado para prever ou projetar o futuro não satisfaz mais as necessidades atuais das empresas. Qualquer desvio significativo em relação às tendências históricas enfraquece a previsão de forma relevante, sendo que tais desvios estão ocorrendo com maior frequência (WRIGHT; KROLL; PARNELL, 2000).

Diante desse contexto, métodos de projeção mais precisos são imprescindíveis. Assim, os estudos de Cenários Prospectivos ganham notoriedade, uma vez que identificam possíveis tendências do ambiente organizacional em um determinado período de tempo, possibilitando a elaboração de estratégias eficazes para se resguardar dos riscos decorrentes de cenários desfavoráveis (SILVA, 2010).

Prospectar cenários, diferentemente do que possa transparecer, não se trata de uma tentativa de prever o futuro ou de adivinhar circunstâncias que estarão presentes no caminho pelo qual a organização pretende seguir. Sua essência é entender, projetar e experimentar diversos futuros, no intuito de traçar estratégias para enfrentar as incertezas, ou seja, as conjunturas não esperadas (RANGEL, 2012).

Na prospecção de futuro no Brasil, devem-se ressaltar como pioneiros e divulgadores sobre a técnica de cenários, os acadêmicos e pesquisadores: Rattner (1979), Jaguaribe (2000), Grumbach (2002), Marcial (2002), Araújo (2001), Bethlem (2002), Porto (2001), Almeida (2002) e Buarque (2003).

Marcial \& Grumbach (2002) escrevem que é relativamente recente no Brasil a prática de elaboração de cenários, sendo que as primeiras empresas a utilizarem tal prática foram o BNDES, a Eletrobrás, a Petrobrás e a Eletronorte, em meados da década de 1980, em função de operarem com projetos de longo período de maturação, o que exigia uma visão de longo prazo.

Uma nova utilização da técnica prospectiva no Brasil foi coordenada pela antiga Secretaria de Assuntos Estratégicos (SAE) da Presidência da República, que iniciou em 1996 os três estudos que geraram, em 1997, 
"Cenários Extrapolatórios do Brasil em 2020" e, em 1998, "Os Cenários desejados para o Brasil" (BRASIL, 2007b).

Em substituição à Secretaria de Assuntos Estratégicos (SAE), foi criado - Núcleo de Assuntos Estratégicos (NAE), vinculado à Casa Civil da Presidência da República, que estruturou o projeto "Brasil em três tempos: 2007, 2015 e 2022"; Metas para o Milênio estabelecidas para cada país signatário no contexto da Organização das Nações Unidas (ONU) e um estimulante estudo prospectivo em relação ao ano em que o Brasil comemorará os 200 anos de independência de Portugal (PNUD, 2007).

Portanto, tem-se um conceito cujo termo remonta à antiguidade, mas que ganha espaço nas organizações a partir da segunda metade do século 20 e com maior ênfase no Brasil entre os séculos 20 e 21.

\subsection{Métodos de Construção de Cenários}

A construção de cenários requer o uso de ferramentas simples, visando facilitar o entendimento (OLIVEIRA; FORTE, 2009) e deve ser escolhida de acordo com a acessibilidade do método conforme a necessidade do cliente (DESTATTE, 2010) para estimular a imaginação e facilitar a apropriação de quem for utilizá-lo (BEZOLD, 2010).

O planejamento de cenários consiste em duas etapas. Na primeira, os cenários são desenvolvidos por meio de um processo sistemático de retratar situações futuras e, na segunda, o planejamento estratégico é baseado no resultado do desenvolvimento de cenários (GRACHT; DARKOW, 2010).

Toda organização é impulsionada por inúmeros fatores-chave, sendo que, identificar e analisar esses fatores são as intenções do método de planejamento por cenários. Uma das maneiras de identificá-los é pelas forças motrizes (sociedade, tecnologia, economia, política e ambiente), que são elementos que direcionam o mapa dos cenários, conduzem e determinam o andamento da história (GODET, 2010).

As variáveis podem originar-se do ambiente interno e externo composto pelas dimensões política, econômica, social e tecnológica (MARTINS et al., 2009). A identificação das variáveis depende dos stakeholders que podem influenciar pelas ações, decisões, políticas, práticas ou objetivos da organização. Os atores podem ser indivíduos e grupos, decisores, organizações ou associações de classes que influenciam ou recebem 
influência significativa do sistema (MARCIAL; GRUMBACH, 2002), e os eventos são situações que podem ocorrer ou uma nova política que será criada (BAÑULS; TUROFF, 2011).

Apesar da extensa variedade de métodos, em geral, eles incluem passos, etapas, fases, análise de variáveis, seleção de atores e elaboração de cenários múltiplos (GODET, 2010).

Basicamente, um cenário completo contempla seis componentes principais: um título, uma filosofia, as variáveis, os atores, as cenas e as trajetórias. Os cenários devem ser possíveis (todos os que a mente humana puder imaginar), realizáveis (todos os possíveis de acontecer e que levam em conta os condicionantes do futuro) e desejáveis (encontram-se em qualquer parte do possível, mas nem todos são realizáveis) (CAVALCANTI, 2001; COATES; DURANCE; GODET, 2010; MARCIAL; GRUMBACH, 2002).

Blanning e Reinig (1998) propõem um método estruturado de avaliação das variáveis nos cenários esboçados, além de discutirem sua implementação por intermédio de um caso. Citados autores sugerem que se faça uma lista de eventos indicando-se a probabilidade de ocorrência destes eventos, obtida a partir da sua análise por um grupo de participantes do processo. Em seguida é construída uma matriz de eventos, onde no eixo horizontal tem-se a probabilidade do evento $(P)$ e no eixo vertical o quanto o evento é favorável ou desfavorável (F) para a organização ou empresa que está desenvolvendo a análise. Propõem, então, a construção de três cenários:

1. Cenário otimista - constituído dos eventos com médias e altas probabilidades de virem ao correr e que sejam mediana ou altamente favoráveis para o setor;

2. Cenário pessimista - constituído pelos eventos que apresentem médias e elevadas probabilidades de virem a ocorrer e que sejam desfavoráveis para o setor;

3. Cenário realista - constituído de todos os eventos que apresentem elevada probabilidade de virem a ocorrer.

As variáveis que possuírem baixa probabilidade e baixa favorabilidade podem ser consideradas varáveis ocultas. No entanto, alguns desses eventos podem ser de interesse por outras razões. Por exemplo, a baixa probabilidade e a baixa favorabilidade podem ser notáveis como prejudicial e improvável, ou seja, quaisquer variáveis podem ser descritas no final dos cenários e até 
serem consideradas variáveis para ser analisada em situações futuras. Não aparecem no cenário pessimista porque são consideradas improváveis de ocorrência.

\section{METODOLOGIA}

\subsection{Tipologia da Pesquisa}

Adotou-se para essa investigação o tipo de pesquisa descritiva, pois se busca descrever os futuros possíveis para os próximos treze anos, sendo os dados coletados por meio de questionários enviados para os especialistas na fase de coleta de dados, contribuindo para a identificação e descrição das variáveis que mais impactam e desenvolvimento dos três cenários prospectados (otimista, pessimista e realista).

Apoia-se, inicialmente, no levantamento de fontes secundárias, levantamento de experiências, levantamento bibliográfico e levantamento de pesquisas já realizadas (SILVERMAN, 2009; MATTAR, 1996). Classifica-se, portanto, como uma pesquisa descritiva, pois se realizou um levantamento na literatura, além de entrevistas com pesquisadores, e posteriormente com executivos estratégicos de UCs, para interpretar a realidade a partir dos dados coletados, com o propósito de identificar variáveis chaves para prospectar cenários. O método de pesquisa descritiva busca a resolução de problemas por meio de entrevistas com peritos para validação de conteúdo, melhorando as práticas, seja pela observação, análise e descrições objetivas (THOMAS; NELSON; SILVERMAN, 2007). A pesquisa descritiva analisa e correlaciona fatos ou fenômenos (variáveis) sem manipulá-los e usa padrões textuais como, por exemplo, questionários para identificação do conhecimento (MALHOTRA, 2005; MATTAR, 1996; RICHARDSON, 1999).

\subsection{PROCEDIMENTO DE COLETA DE DADOS E AMOSTRA}

Inicialmente foi realizada a coleta de dados por meio da leitura de artigos acadêmicos, a fim de obter uma base sólida e consolidada de eventos que pudessem ajudar a compreender os cenários, seguido de uma análise de conteúdo de cada um dos eventos, em que os eventos iguais, eventos parecidos e eventos inclusivos se tornaram um único evento e os eventos diferentes foram avaliados e mantidos (BARDIN, 2011). Posteriormente, foi realizado um pré-teste com o envio do questionário para quatro especialistas 
(professores doutores) no intuito de obter feedback sobre a sua clareza de propósito, sua lógica e adequação de vocabulário (MALHOTRA, 2002).

Com o objetivo da construção de cenários voltados à UC no Brasil, para o horizonte temporal de 2017 a 2030, esta pesquisa prospectou a opinião de 15 especialistas em EC e executivos de UCs, a fim de identificar suas percepções do futuro da Universidade Corporativa no Brasil. Assim, buscouse o contato dos responsáveis pelas Universidades Corporativas de empresas e também de especialistas no assunto e que tivessem trabalho científico publicado. Dentre os 99 questionários encaminhados, 15 responderam à pesquisa, sendo os resultados apresentados na seção Resultados e Discussão.

\subsection{MÉTODO PARA A CONSTRUÇÃO DE CENÁRIOS PROSPECTIVOS}

O trabalho foi efetuado com a utilização da metodologia sugerida por Blanning e Reinig (1998), apoiada pelo método de Marcial e Grumbach (2002), estando dividido em cinco fases:

Fase 1: retirada da literatura uma lista de eventos e, posteriormente, validada por quatro peritos.

Fase 2: realizada uma pesquisa para desenvolvimento de uma Matriz de Probabilidade e Favorabilidade por meio de uma lista de eventos, que consiste na ótica dos especialistas sobre o impacto gerado para as UCs no Brasil.

Fase 3: a matriz foi submetida a especialistas e executivos de UCs para que indicassem a probabilidade de ocorrência e a favorabilidade e a pertinência dos eventos caso ocorressem, de acordo escala demonstrada na Tabela 1.

\section{Tabela 1 -Escala classificatória de Pertinência segundo o método Marcial e Grumbach (2002), e Probabilidade e Favorabilidade de acordo com Blanning e Reinig (1998)}

\begin{tabular}{l|l|l}
\hline \multicolumn{1}{c|}{ Pertinência: Escala 1 a 9 } & $\begin{array}{c}\text { Probabilidade: Escala 0\% } \\
\text { a } \mathbf{1 0 0 \%}\end{array}$ & $\begin{array}{c}\text { Favorabilidade: Escala } \\
\mathbf{0} \text { a } \mathbf{1 0}\end{array}$ \\
\hline 1 = Sem pertinência & Zero = não acontecerá. & $0=$ não favorável. \\
\hline 5 = Razoável pertinência & $50 \%=$ metade da chance. & $5=$ favorabilidade neutra. \\
\hline $9=$ Extrema pertinência & $100 \%=$ certeza & $10=$ muito favorável. \\
\hline
\end{tabular}

Fonte: Adaptado de Blanning e Reinig (1998) e Marcial e Grumbach (2002).

Fase 4: realizada a análise de cenários baseada nos fatores e na lista de eventos analisados pelos especialistas entrevistados. Dessa forma, torna-se possível a visualização e a interação de componentes que agregam em conjuntos complexos, além do entendimento da multiplicidade e interdependência das 
causas e variáveis dos problemas, bem como a antecipação de possíveis tendências de soluções.

A pertinência dos eventos foram avaliadas por meio da média ponderada. Assim, as frequências vezes os pesos de 1 a 9, foram divididas por 135, o que corresponde a 9 (pertinência máxima) x 15 (frequência máxima, ou seja, o total de respondentes). Selecionou-se os eventos a partir da média ponderada de 0,70, totalizando 22 Eventos.

O trabalho segue a proposta metodológica de Blanning e Reinig (1998) de construção de cenários, aprofundando o processo de tratamento dos dados proposto por aqueles autores, sugerindo a abordagem sistêmica como forma de lidar com esta complexidade. Assim, são possíveis a visualização e a interação de componentes que agregam em conjuntos complexos, além do entendimento da multiplicidade e interdependência das causas e variáveis dos problemas, bem como a antecipação de possíveis tendências de soluções.

Ressalta-se que foi utilizado o modelo seminal de Blanning e Reinig intitulado Building Scenarios for Hong Kong Using SEM (1998), que também foi utilizado em outros 34 trabalhos acadêmicos, pois apresenta uma metodologia que propõe um método estruturado de avaliação dos eventos nos cenários esboçados e discutem sua implementação por meio de um caso e ainda sugerem a realização de uma lista de eventos indicando a probabilidade e a favorabilidade com a elaboração de uma matriz de eventos e a construção de três cenários.

\section{RESULTADOS E ALINHAMENTO TEÓRICO}

Esta seção apresenta a análise dos dados da pesquisa dividida em três subseções. Na primeira, estão a identificação dos eventos obtidos por meio da leitura de artigos acadêmicos e a validação por especialistas. Na segunda subseção, encontra-se a exclusão das variáveis para composição dos cenários prospectivos. Por fim, na terceira e última subseção, há a descrição dos três cenários: otimista, pessimista e realista conforme modelo proposto pelos autores Blanning e Reinig (1998).

\subsection{Identificação Dos Eventos}

O modelo sugerido por Blanning e Reinig (1998) pressupõe a elaboração de três cenários, sendo que cada qual abriga uma quantidade de eventos de 
acordo com sua Probabilidade e Favorabilidade. Os cenários foram dispostos em um plano cartesiano, comporto por eixos $X$ (dimensionador de Probabilidade) e Y (dimensionador de Favorabilidade).

$\mathrm{Na}$ Tabela 2, a seguir, podem ser observados todos os eventos e os resultados da aplicação do método utilizado.

Tabela 2 - Matriz de pertinência, probabilidade e favorabilidade dos eventos: cenários prospectivos para as s (UCs) no Brasil

\begin{tabular}{|c|c|c|c|c|c|c|c|c|c|c|c|c|c|}
\hline \multirow[t]{2}{*}{ No } & \multirow[t]{2}{*}{ Descrição } & \multicolumn{10}{|c|}{ Pertinência } & \multirow{2}{*}{$\begin{array}{c}\text { Probab. } \\
\text { Média }\end{array}$} & \multirow{2}{*}{$\begin{array}{l}\text { Favorab. } \\
\text { Média }\end{array}$} \\
\hline & & 1 & 2 & 3 & 4 & 5 & 6 & 7 & 8 & 9 & MP & & \\
\hline $\mathrm{E} 1$ & $\begin{array}{l}\text { Ter que cumprir uma nova } \\
\text { legislação especifica para UCs }\end{array}$ & 6 & 1 & 1 & 0 & 0 & 2 & 1 & 2 & 2 & 0,47 & $38 \%$ & $39 \%$ \\
\hline E2 & $\begin{array}{l}\text { Instabilidade Política pode } \\
\text { afetar os investimentos }\end{array}$ & 1 & 0 & 1 & 0 & 1 & 0 & 5 & 2 & 5 & 0,77 & $74 \%$ & $39 \%$ \\
\hline E3 & $\begin{array}{l}\text { Instabilidade Econômica pode } \\
\text { afetar os investimentos }\end{array}$ & 0 & 0 & 0 & 0 & 1 & 0 & 1 & 4 & 9 & 0,92 & $80 \%$ & $47 \%$ \\
\hline E4 & 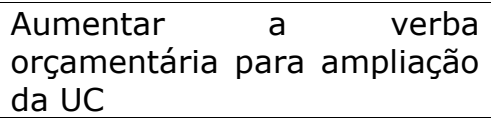 & 1 & 0 & 1 & 0 & 0 & 0 & 6 & 3 & 4 & 0,78 & $59 \%$ & $68 \%$ \\
\hline E5 & $\begin{array}{l}\text { Aumentar as parcerias com as } \\
\text { Instituições de Ensino } \\
\text { Superior - IES }\end{array}$ & 0 & 0 & 0 & 1 & 0 & 3 & 2 & 4 & 5 & 0,83 & $75 \%$ & $78 \%$ \\
\hline E6 & $\begin{array}{l}\text { Maior entendimento do } \\
\text { negócio pelo RH }\end{array}$ & 0 & 0 & 0 & 1 & 1 & 0 & 1 & 2 & 10 & 0,90 & $75 \%$ & $79 \%$ \\
\hline E7 & $\begin{array}{l}\text { Avaliar o ROI do treinamento } \\
\text { com base nos resultados } \\
\text { obtidos com a aplicação do } \\
\text { aprendizado }\end{array}$ & 1 & 0 & 0 & 0 & 2 & 1 & 4 & 0 & 7 & 0,80 & $59 \%$ & $69 \%$ \\
\hline E8 & $\begin{array}{l}\text { Aumentar a média geral de } \\
\text { horas de treinamento }\end{array}$ & 0 & 2 & 2 & 0 & 2 & 2 & 3 & 1 & 3 & 0,65 & $64 \%$ & $59 \%$ \\
\hline E9 & $\begin{array}{lccc}\text { Aumentar } & 0 & \text { Ensino } & \text { à } \\
\text { Distância - EAD } & & \\
\end{array}$ & 0 & 0 & 0 & 0 & 0 & 1 & 3 & 6 & 5 & 0,89 & $85 \%$ & $83 \%$ \\
\hline E10 & $\begin{array}{l}\text { Aumentar a quantidade de } \\
\text { empresa com estrutura } \\
\text { própria de UC }\end{array}$ & 1 & 0 & 0 & 1 & 2 & 2 & 3 & 2 & 4 & 0,74 & $58 \%$ & $65 \%$ \\
\hline E11 & $\begin{array}{l}\text { Consorciar-se com empresas } \\
\text { não concorrentes que } \\
\text { compartilham necessidades e } \\
\text { treinamentos para reduzir } \\
\text { custos }\end{array}$ & 1 & 0 & 0 & 0 & 1 & 1 & 4 & 1 & 7 & 0,82 & $66 \%$ & $74 \%$ \\
\hline E12 & $\begin{array}{l}\text { Maior competição das UCs } \\
\text { com as universidades } \\
\text { tradicionais }\end{array}$ & 5 & 0 & 0 & 3 & 1 & 2 & 3 & 0 & 1 & 0,47 & $33 \%$ & $45 \%$ \\
\hline E13 & $\begin{array}{l}\text { Configurar a UC como } \\
\text { instituição de ensino regular } \\
\text { (faculdade ou universidade) }\end{array}$ & 5 & 2 & 1 & 2 & 0 & 0 & 1 & 2 & 2 & 0,45 & $49 \%$ & $35 \%$ \\
\hline E14 & $\begin{array}{l}\text { Aumento das universidades } \\
\text { setoriais fundadas a partir de } \\
\text { parcerias entre empresas do } \\
\text { mesmo segmento de atuação }\end{array}$ & 1 & 0 & 0 & 0 & 1 & 2 & 5 & 2 & 4 & 0,78 & $65 \%$ & $71 \%$ \\
\hline E15 & $\begin{array}{l}\text { Aumentar os treinamentos } \\
\text { online como alternativa para } \\
\text { o pequeno negócio }\end{array}$ & 0 & 1 & 1 & 1 & 1 & 0 & 2 & 2 & 7 & 0,79 & $73 \%$ & $77 \%$ \\
\hline E16 & $\begin{array}{l}\text { Aumento do surgimento de } \\
\text { UCs em empresas de médio e } \\
\text { pequeno portes }\end{array}$ & 1 & 0 & 3 & 0 & 3 & 2 & 2 & 3 & 1 & 0,62 & $62 \%$ & $56 \%$ \\
\hline
\end{tabular}


Tabela 2 - Matriz de pertinência, probabilidade e favorabilidade dos eventos: cenários prospectivos para as s (UCs) no Brasil

\begin{tabular}{|c|c|c|c|c|c|c|c|c|c|c|c|c|c|}
\hline \multirow[t]{2}{*}{$\mathrm{N}^{\circ}$} & \multirow[t]{2}{*}{ Descrição } & \multicolumn{10}{|c|}{ Pertinência } & \multirow{2}{*}{$\begin{array}{l}\text { Probab. } \\
\text { Média }\end{array}$} & \multirow{2}{*}{$\begin{array}{l}\text { Favorab. } \\
\text { Média }\end{array}$} \\
\hline & & 1 & 2 & 3 & 4 & 5 & 6 & 7 & 8 & 9 & MP & & \\
\hline E17 & $\begin{array}{l}\text { Emitir diploma pela própria } \\
\text { instituição em parceria com o } \\
\text { sistema nacional de educação }\end{array}$ & 3 & 2 & 1 & 1 & 1 & 0 & 2 & 3 & 2 & 0,55 & $57 \%$ & $49 \%$ \\
\hline E18 & $\begin{array}{l}\text { Oferecer cursos sem garantia } \\
\text { de praticar o que aprendeu }\end{array}$ & 7 & 2 & 4 & 0 & 0 & 0 & 1 & 0 & 1 & 0,29 & $33 \%$ & $25 \%$ \\
\hline E19 & $\begin{array}{l}\text { Permitir que funcionários } \\
\text { decidam quais cursos farão }\end{array}$ & 1 & 0 & 2 & 1 & 1 & 3 & 3 & 1 & 3 & 0,67 & $68 \%$ & $62 \%$ \\
\hline E20 & $\begin{array}{l}\text { Capacitar com maior ênfase a } \\
\text { média e alta gerenciais }\end{array}$ & 1 & 0 & 0 & 0 & 2 & 0 & 4 & 0 & 8 & 0,82 & $79 \%$ & $83 \%$ \\
\hline E21 & $\begin{array}{l}\text { Desenvolver as lideranças das } \\
\text { empresas }\end{array}$ & 0 & 0 & 0 & 0 & 1 & 1 & 1 & 2 & 10 & 0,92 & $86 \%$ & $92 \%$ \\
\hline E22 & $\begin{array}{l}\text { Ter resultados voltados para } \\
\text { aspectos sociais e ambientais, } \\
\text { com pouco enfoque } \\
\text { acadêmico }\end{array}$ & 2 & 1 & 0 & 0 & 1 & 0 & 6 & 2 & 3 & 0,69 & $61 \%$ & $57 \%$ \\
\hline E23 & $\begin{array}{l}\text { Estar próximo do centro de } \\
\text { poder, com o engajamento da } \\
\text { Alta Administração }\end{array}$ & 0 & 0 & 0 & 0 & 0 & 1 & 1 & 3 & 10 & 0,94 & $78 \%$ & $87 \%$ \\
\hline E24 & $\begin{array}{l}\text { Contemplar a capacitação de } \\
\text { aposentados } \\
\text { funcionários) }\end{array}$ & 3 & 1 & 2 & 3 & 1 & 1 & 2 & 0 & 2 & 0,49 & $47 \%$ & $45 \%$ \\
\hline E25 & $\begin{array}{lr}\begin{array}{l}\text { Aumento da } \\
\text { novas }\end{array} & \text { exploração de } \\
\text { (gamificaçãa, } & \text { infográficostas, } \\
\text { animação e flash e simulações } \\
\text { de curta duração) pelas UCs }\end{array}$ & 0 & 0 & 0 & 0 & 1 & 1 & 1 & 3 & 9 & 0,91 & $82 \%$ & $80 \%$ \\
\hline E26 & $\begin{array}{l}\text { Aumento do investimento em } \\
\text { linguagens novas e recursos } \\
\text { tecnológicos que possibilitem } \\
\text { aos funcionários acesso aos } \\
\text { conteúdos via tablet e } \\
\text { smartfones }\end{array}$ & 0 & 0 & 0 & 0 & 0 & 0 & 2 & 3 & 10 & 0,95 & $85 \%$ & $87 \%$ \\
\hline E27 & 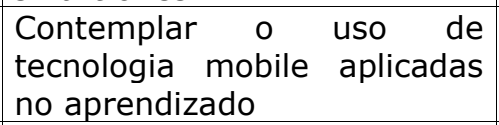 & 0 & 0 & 0 & 0 & 0 & 0 & 1 & 5 & 9 & 0,95 & $87 \%$ & $90 \%$ \\
\hline E28 & $\begin{array}{l}\text { Envolver } \\
\text { fornecedores, terceiros es, } \\
\text { comunidade }\end{array}$ & 0 & 0 & 0 & 0 & 1 & 2 & 3 & 1 & 8 & 0,87 & $71 \%$ & $74 \%$ \\
\hline E29 & $\begin{array}{l}\text { Aumentar os hard skills em } \\
\text { comparação aos soft skills }\end{array}$ & 1 & 2 & 1 & 1 & 3 & 1 & 3 & 2 & 1 & 0,58 & $48 \%$ & $46 \%$ \\
\hline E30 & $\begin{array}{lll}\text { Reduzir o tempo } & \text { dos } \\
\text { treinamentos por meio da } & \text { por } \\
\text { fragmentação dos conteúdos }\end{array}$ & 1 & 1 & 0 & 0 & 1 & 3 & 4 & 2 & 3 & 0,72 & $71 \%$ & $69 \%$ \\
\hline E31 & $\begin{array}{l}\text { Aumento do uso do peer to } \\
\text { peer learning }\end{array}$ & 0 & 0 & 0 & 0 & 0 & 0 & 7 & 2 & 6 & 0,89 & $76 \%$ & $81 \%$ \\
\hline E32 & $\begin{array}{l}\text { Aumentar as equipes com } \\
\text { dedicação exclusiva as UCS }\end{array}$ & 1 & 1 & 2 & 0 & 1 & 2 & 3 & 2 & 3 & 0,66 & $54 \%$ & $65 \%$ \\
\hline E33 & $\begin{array}{ll}\text { Institucionalizar } & \text { os } \\
\text { mecanismos de avaliação de } \\
\text { resultados das UCs }\end{array}$ & 0 & 0 & 1 & 0 & 2 & 3 & 2 & 2 & 5 & 0,78 & $70 \%$ & $76 \%$ \\
\hline E34 & $\begin{array}{lcr}\text { Aumento das UCs em países } \\
\text { emergentes } & \text { e } & \text { em } \\
\text { organizações } & \text { pequenas } & \text { e } \\
\text { médias } & & \\
\end{array}$ & 4 & 0 & 0 & 0 & 2 & 5 & 2 & 1 & 1 & 0,55 & $37 \%$ & $54 \%$ \\
\hline E35 & $\begin{array}{l}\text { Aumento da capacidade dos } \\
\text { indivíduos promover o bom } \\
\text { senso coletivo. }\end{array}$ & 0 & 1 & 0 & 0 & 0 & 1 & 3 & 4 & 4 & 0,83 & $71 \%$ & $71 \%$ \\
\hline
\end{tabular}


Tabela 2 - Matriz de pertinência, probabilidade e favorabilidade dos eventos: cenários prospectivos para as s (UCs) no Brasil

\begin{tabular}{|c|c|c|c|c|c|c|c|c|c|c|c|c|}
\hline \multirow[t]{2}{*}{ No } & \multirow[t]{2}{*}{ Descrição } & \multicolumn{9}{|c|}{ Pertinência } & \multirow{2}{*}{$\begin{array}{c}\text { Probab. } \\
\text { Média }\end{array}$} & \multirow{2}{*}{$\begin{array}{l}\text { Favorab. } \\
\text { Média }\end{array}$} \\
\hline & & 1 & 2 & 3 & \begin{tabular}{l|l}
4 & 5 \\
\end{tabular} & 6 & 7 & 8 & 9 & MP & & \\
\hline E36 & Outros & 5 & 1 & $\overline{0}$ & 0 & 0 & 0 & 3 & $\overline{4}$ & & $50 \%$ & \\
\hline
\end{tabular}

Fonte: Elaborada pelos autores (2017).

Cabe destacar que foi realizada uma tabulação por meio do uso do Software Microsoft Excel, em que, de acordo com o apurado, determinaramse algumas premissas (SILVA; SANTOS, 2015), sendo:

- Cenário Otimista (Probabilidade acima de 70\% e Favorabilidade acima de 65\%);

- Cenário Pessimista (Probabilidade acima de 70\% e a Favorabilidade menor que $45 \%$ ) e

- Cenário Realista (Probabilidade acima de 75\% e com qualquer Favorabilidade.

Os 36 eventos para a construção dos cenários (otimista, realista e pessimista) encontram-se demonstrados no Gráfico 1, a seguir:

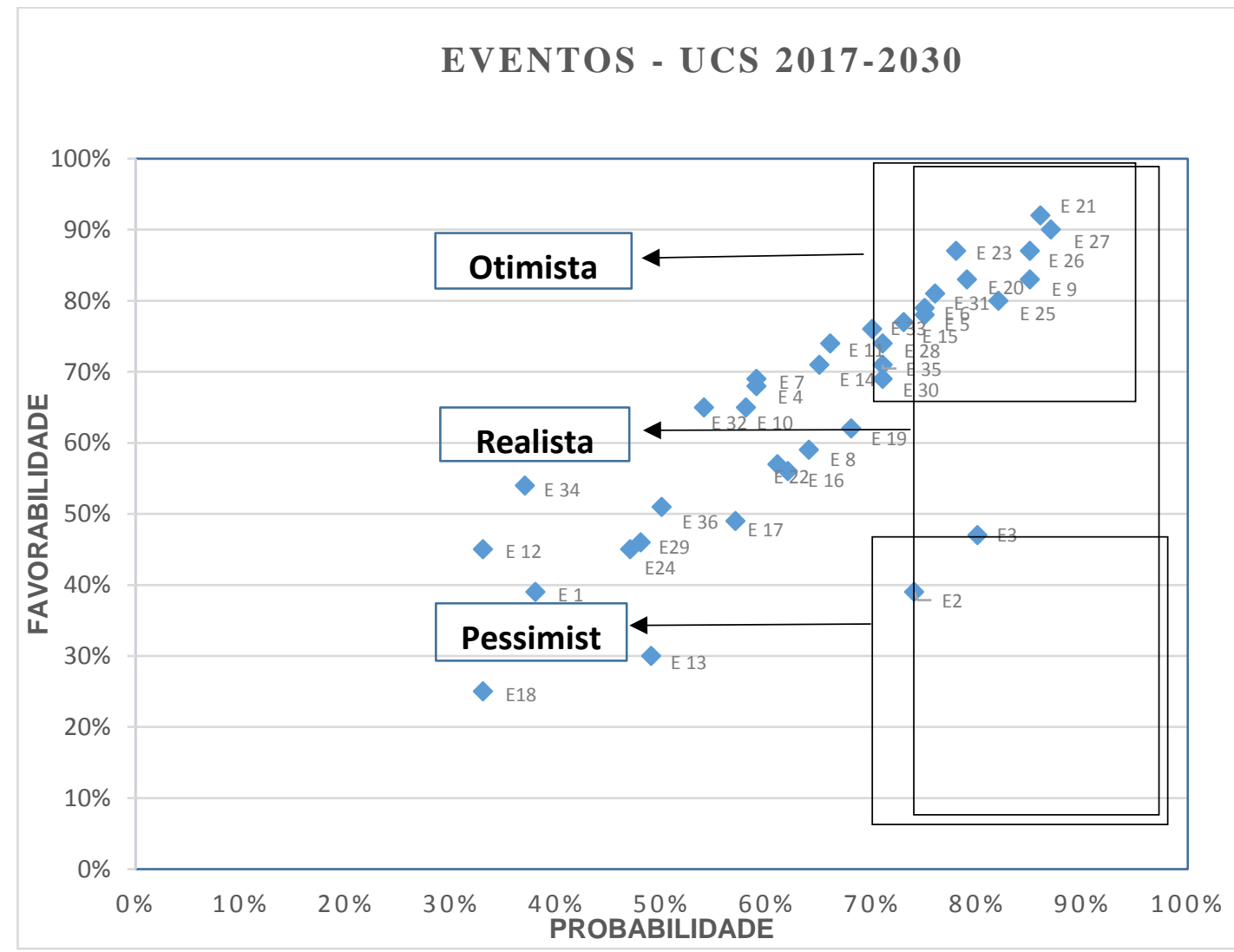

Gráfico 1: Eventos para construção de cenários

Fonte: Elaborado pelos autores (2017). 
Observa-se no Gráfico 1, que a maioria dos eventos possui alta probabilidade e favorabilidade, englobando os 15 eventos considerados otimistas e realistas, havendo apenas um evento pessimista.

Já os demais 20 eventos identificados abaixo desta métrica, não apareceram em nenhum dos cenários, considerados eventos de baixa ou média probabilidade e favorabilidade, conforme estabelece a metodologia de Blanning e Reinig (1998). Entretanto, alguns desses eventos podem ser de interesse por outras razões como, por exemplo, podem ser potencialmente prejudiciais e ou improváveis.

\subsection{Cenários Prospectivos Para As UCS Brasileiras}

Esta subseção apresenta os cenários prospectados para que as UCs brasileiras fiquem atentas para o horizonte 2030. Assim, prospectam-se os três cenários a seguir:

\subsubsection{Cenário Otimista}

O Cenário otimista nesse estudo é composto pelas variáveis que apresentam elevada probabilidade de ocorrência e que são muito favoráveis para as UCs brasileiras. Este cenário é composto por 15 dos 16 eventos: (E3); (E5); (E6); (E9); (E15); (E20); (E21); (E23); (E25); (E26), (E27); (E28); (E30); (E31) e (E35). Destes, apenas seis eventos (E5), (E6), (E15), (E28), $(E 30)$ e (E35) são apenas otimistas, pois apresentam probabilidade menor que $75 \%$ e maior que $70 \%$, apesar de muito próximo.

No caso em questão, todos oito dos 15 eventos encontram-se em ambos os cenários (otimista e realista), pois apresentam uma elevada possibilidade de ocorrência. Para o cenário otimista, têm-se dois eventos empatados tecnicamente, uma vez que o Evento 21 (Desenvolver as lideranças da Empresa) (BARBOSA, 2016; CASTRO; EBOLI, 2013); GLOBALCCU, 2017); MARINELLI, 2013) e o Evento 27 (Contemplar o uso de tecnologia mobile aplicadas no aprendizado) (ABEL; LI, 2012); CASTRO; EBOLI, 2013); GLOBALCCU, 2017) são os eventos que indicam o maior percentual de probabilidade de ocorrência e favorabilidade, respectivamente, com $86 \%$ e $92 \%$ e $87 \%$ e $90 \%$. É uma razão de grande representatividade para o futuro das Universidades Corporativas, uma vez que ter capital humano capacitado e tecnologia mobile disponível são fundamentais para o futuro das UCs. 
O Evento 9 (Aumentar o Ensino à Distância), juntamente com o Evento 25 (Aumento da exploração de novas ferramentas, como "gamificação", infográficos, animação e flash e simulações de curta duração pelas UCs e o Evento 26 (Aumento do investimento em linguagens novas e recursos tecnológicos que possibilitem aos funcionários acesso aos conteúdos via tablet e smartfones), apoiados em Barbosa (2016) e Delloite (2016), com probabilidade entre 82 e $85 \%$ e favorabilidade entre 80 e $87 \%$, contemplam assuntos muito similares que envolvem o uso de tecnologias que tornem os cursos mais atrativos e acessíveis, bem como que possibilitem envolver mais pessoas interessadas com maior rapidez e menores custos, possibilitando, consequentemente, a ampliação do negócio UC.

Salienta-se, ainda, que os demais nove eventos que fazem parte do cenário otimista, sendo: Evento 5 (Aumento das parcerias com IES) (GLOBALCCU (2017), Evento 6 (Maior entendimento do negócio pelo RH) (MARINELLI, 2013), Evento 15 (Aumentar os treinamentos online)(BARBOSA 2016; DELLOITE, 2016), Evento 20 (Capacitar com maior ênfase a média e alta gerências (BARBOSA, 2016; CASTRO; EBOLI, 2013); GLOBALCCU, 2017); MARINELLI, 2013), Evento 23 (Estar próximo do centro de poder) (CASTRO; EBOLI, 2013), Evento 28 (Envolver clientes, fornecedores, terceiros e comunidade) (BARBOSA, 2016); CASTRO; EBOLI, 2013); FREITAS-DIAS; ALBUQUERQUE, 2014); GUERCI; BARTEZZAGHI; SOLARI, 2010); MARINELLI, 2013); PARKER, 2017), Evento 30 (Reduzir o tempo dos treinamentos por meio da fragmentação dos conteúdos) (Peritos), Evento 31 (Aumento do uso do peer to peer learning) (Peritos) e Evento 35 (Aumento da capacidade dos indivíduos promoverem o bom senso coletivo) (Peritos) são, de fato, eventos muito importantes e apresentam uma alta pertinência média $(80 \%)$, uma probabilidade média de ocorrência relevante (74\%) e uma favorabilidade média elevada (78\%), o que demonstra que tais eventos são extremamente relevantes, sendo de grande importância até mesmo para o futuro das Universidades Corporativas brasileiras.

\subsubsection{Cenário Realista}

O Cenário realista é composto pelas variáveis que apresentam alta probabilidade de ocorrência, independentemente de sua favorabilidade para as Universidades Corporativas brasileiras. Este cenário é composto por $57,1 \%$ dos 
eventos do cenário otimista, com exceção dos eventos (E5); (E8); (E15); (E28); (E30) e (E35), pois apresentam probabilidade sensivelmente abaixo de $75 \%$, respectivamente, $73 \%, 71 \%, 71 \%$ e 71\%, independentemente da favorabilidade. Também é composto pelo Evento 3 (Instabilidade Econômica pode afetar os investimentos) (Peritos), uma vez que possui $80 \%$ de probabilidade de ocorrer.

Em relação à Instabilidade Econômica afetar os investimentos, observa-se que $60 \%$ dos respondentes indicaram que possui alta pertinência, além de ter $80 \%$ de probabilidade de impactar os investimentos em Universidades Corporativas, sendo, portanto, desfavorável para a construção de cenários.

\subsubsection{Cenário Pessimista}

O Cenário pessimista é composto por eventos que apresentam elevada probabilidadede ocorrência (maior que 70\%) e que tenham baixa favorabilidade (menor que 45\%), ou seja, denotem ser desfavoráveis para as UCs brasileiras. Como se pode verificar no Gráfico 1, há apenas um evento E2 (Instabilidade Política pode afetar os investimentos (Peritos).

O Evento 2 se trata de uma variável exógena e apresenta favorabilidade baixa $(39 \%)$ e alta probabilidade de ocorrência (74\%), sendo muito desfavorável para as Universidades Corporativas brasileiras. Trata-se de um evento diretamente influenciado pelas ações governamentais.

A tendência para o controle e estabilização do Evento acima pode favorecer o processo de consolidação e contribuir para viabilizar as UCs brasileiras.

\section{CONSIDERAÇÕES FINAIS}

A partir da pesquisa foi possível prospectar e construir três cenários, a partir dos eventos que poderão vir a impactar as UCs no período de tempo considerado, sendo necessárias a adoção de estratégias eficazes e desenvolvimento de recursos para se enfrentar as incertezas e resguardar dos riscos decorrentes de cenários desfavoráveis.

Dentro do Cenário Otimista foi possível verificar a apresentação de $87,5 \%$ dos eventos (14 de 16 ), sendo que a grande parte destes $(57,1 \%)$ também foi observada no Cenário Realista (8 de 14). Já o Cenário Pessimista possui apenas $6,25 \%$ dos eventos levantados ( 1 de 16 ). 
Merecem destaque o Evento 21 (Desenvolver as lideranças da Empresa) e o Evento 27 (Contemplar o uso de tecnologia mobile aplicadas no aprendizado), pois são as variáveis que indicam o maior percentual de probabilidade de ocorrência e favorabilidade, todas acima de $87 \%$, sendo a questão da liderança fator crucial para a continuidade das empresas, assim como o uso da tecnologia móvel no aprendizado imprescindível para o futuro das Universidades Corporativas.

Questões políticas e econômicas se mostram com alta probabilidade, mas baixa favorabilidade para as UCs, enquadrando-se no Cenário Pessimista. Entende-se tal resultado como reflexo do que vem ocorrendo com o Brasil em especial em 2017 com a deflagração de inúmeros casos de corrupção envolvendo grande parte da classe política brasileira, assim como a grave situação econômica dos governos (Federal, Estadual e Municipal) e União decorrente da má gestão e desvios de recursos públicos para outras finalidades, assim como o agravamento da situação econômico-financeira das instituições privadas que tiveram queda de faturamento decorrentes, entre outros, de altos índices de desemprego e também pela insegurança causada pela crise política, aliado a fragilidade dos controles internos destas.

Assim, oportunidades e ameaças que podem estar presentes no ambiente de negócios das UCs brasileiras foram identificadas, mapeadas e analisadas por meio da prospecção de cenários, permitindo enfrentar as incertezas e resguardar-se dos principais riscos do negócio.

A prospecção de Cenários traz às UCs brasileiras bases mais sólidas para compor suas estratégias em médio e longo prazos, reduzindo a vulnerabilidades das UCs.

A aplicabilidade do estudo de Cenários ocorre, especialmente, em condições de incerteza e complexidade como a atual, o que permite adotar uma metodologia que contempla questões operacionais na prospecção de cenários.

Os resultados apontaram 16 variáveis relevantes, consideradas chave, que resultaram em dois cenários prospectivos: um otimista/realista e um pessimista. Salienta-se a grande relevância dos eventos que compõem os cenários otimistas/realistas, totalizando $93,75 \%$ (15 de 16) e apenas 6,25\% de eventos pessimistas (1 de 16) relacionados à variável exógena (política).

Como limitação da pesquisa, preconiza-se que se poderia ter tido mais repostas dos peritos, entretanto, com os quatro peritos que ajudaram na construção dos eventos, totalizam 19 peritos participantes nesta pesquisa. 
Como sugestão para os próximos estudos, podem ser contempladas todas as empresas brasileiras (públicas ou privadas) que possuem UCs (estimada em 600), visando mapear as estratégias, recursos e desempenhos esperados pelas UCs diante dos cenários aqui prospectados e ainda, rever os cenários tendo uma composição de mais especialistas.

\section{REFERÊNCIAS}

$A B E L, A . L . ; L I, \quad J$. Exploring the corporate university phenomenon: Development and implementation of a comprehensive survey. Human Resource Development Quarterly, v. 23, n. 1, 2012, p. 103-128

ALLEN, M.; MCGEE, P. Measurement and evaluation in corporate universities. New Directions for Institutional Research, [S. I. ], n.124, Winter 2004, p. 81-92.

ALPERSTEDT, C. Universidades corporativas: discussão e proposta de uma definição. RAC - Revista de Administração Contemporânea, v. 5, n. 3, p. 149-166, set./dez. 2001.

BAÑULS, V. A.; TUROFF, M. Scenario construction via Delphi and crossimpact analysis, Technological Forecasting \& Social Change, v. 78, n. 9, p. 1579-1602, 2011.

BARBOSA, J. A. S. Universidades corporativas e vantagens competitivas: um estudo multicaso em franquias brasileiras. Tese (Doutorado). São Paulo: Universidade Corporativa, 2016

BARDIN, L. Análise de conteúdo. Trad. Luís Antero Reto e Augusto Pinheiro. São Paulo: Edições 70, 2011.

BEZOLD, C. Lessons from using scenarios for strategic foresight. Technological Forecasting \& Social Change, v. 77, n. 9, p. 1513-1518, 2010. 1964.

BERGER, G. Phénoménologie du temps et prospective. Paris: PUF,

BLANNING, R. W.; REINIG, B. A. Building Scenarios for Hong Kong Using EMS. Long Rang Planning, v. 31, n.6, p. 900-910, 1998.

BRASIL. Secretaria de Planejamento de Longo Prazo. Histórico de planejamento de longo prazo no Brasil. 2007b. Disponível em: <http://www.splp.gov.br/splp_hstorico_planejamento.html>. Acesso em: 20 de out. 2007b.

CASTRO, C. de M.; EBOLI, M. Universidade corporativa: gênese e questões críticas rumo à maturidade. São Paulo, Revista de Administração de Empresas - RAE, São Paulo, v. 53, n.4, jul-ago, 2013.

CAVALCANTI. M. Gestão estratégica de negócios: evolução, cenários, diagnóstico e ação. São Paulo: Thomson Learning, 2001. 
COATES, J.; DURANCE, P.; GODET, M. Strategic Foresight Issue: introduction. Technological Forecasting \& Social Change, v. 77, n. 9, p. 1423-1425, 2010.

DELLOITE. Investimento em educação corporative desponta como alternative para melhorar a qualificação de profissionais - 2016. Disponível em

https://www2.deloitte.com/br/pt/footerlinks/pressreleasespage/pesquisaeducacao-corporativa-no-brasil.html>. Acessado em 15.10.2017.

DESTATTE, P. Foresight: A major tool in tackling sustainable development. Technological Forecasting \& Social Change, v. 77, n. 9, p. 1575-1587, 2010.

DURANCE, P.; GODET, M. Scenario building: uses and abuses. Technological Forecasting \& Social Change, v. 77, n. 9, p. 1488-1492, 2010.

EBOLI, M. Educação Corporativa no Brasil: mitos e verdades. São Paulo: Gente, 2004.

EBOLI, M. Educação Corporativa e desenvolvimento de competências. In: DUTRA J. S.; FLEURY, M. T. L.; RUAS, R. L. Competências: conceitos, métodos e experiências. São Paulo: Atlas, 2008.

EBOLI, M. Uma Revolução Invisível. Harvard Business Review (S.I.), p. 74-85, jun. 2012.

FREITAS-DiAS, C. A. de; ALBUQUeRQUE, L. G. de. Panorama de avaliação de Resultados em educação corporativa no Brasil, R. Adm. Faces Journal, Belo Horizonte, v. 13, n. 1, p. 103-123, jan/mar, 2014.

FREITAS FILHO, H.; OLIVEIRA, M.; SACCOL, A. Z. MOSCAROLA, J. O método de pesquisa survey. Revista de Administração da USP, São Paulo, v. 35, n. 3, p. 105-112, jul./set. 2013.

GLOBALCCU 2017. CCU Awards.Disponível em: <http://www.globalccu.com/>. Acessado em 28.07.2017

GODET, M. Manual de prospectiva estratégica: da antecipação a ação. Lisboa: Dom Quichote, 1993.

GODET, M.; ROUBELAT, F. Scenario Planning: An Open Future. Technological Forecasting and Social Change. v. 65, n.1, p. 1-124, 2000.

GODET, M. Future memories. Technological Forecasting \& Social Change, v. 77, n. 9, p. 1457-1463, 2010.

GRACHT, H. A. V. D.; DCKIOL, I. L. Scenarios for the logistics services industry: A Delphi-based analysis for 2025. International Journal of Production Economics, v. 127, n. 1, p. 46-59, 2010.

GUERCI, M; BARTEZZAGHI,E.; SOLARI, L. Training evaluation in Italian corporate universities: a stakeholder-based analysis, International Journal of Training and Development, v. 14, n. 4, 2010 
HEIJDEN, K. V. D. Planejamento de cenários: a arte da conversação estratégica. Porto Alegre: Bookman, 2004.

KAHN, H. A escalada, metáforas e cenários. Rio de Janeiro: Bloch, 1969.

KIRKPATRICK, D. L.; KIRKPATRICK, J. D. Como avaliar programas de treinamento de equipes: os quatro níveis. Tradução José Henrique Lamensdorf. Rio de Janeiro: Senac Rio, 2010.

MALHOTRA, N. K. et al. Introdução à pesquisa de marketing. São Paulo: Pearson Prentice Hall, 2005.

MARCIAL, E. C.; GRUMBACH, R. J. S. Cenários prospectivos: como construir um futuro melhor. Rio de Janeiro: FGV, 2002.

MARINELLI, M. Autoavaliação institucional: estudo de sistemas de educação corporativa bancos de desenvolvimento brasileiros. (Tese de doutorado). Fortaleza: UFC, 2013

MARTINS, P. P. P.; BOAVENTURA, J. M. G.; COSTA, B. K.; SPERS, R. G.; FISCHMANN, A. A. Cenários para o setor de transportes rodoviários de cargas no Brasil. In: ENCONTRO DE ESTUDOS ESTRATÉGICOS, 4., 2009, Recife. Anais... Recife: ANPAD, 2009.

MATITZ, Q. R. S.; BULGACOV, S. O Conceito Desempenho em Estudos Organizacionais e Estratégia: um Modelo de Análise Multidimensional. RAC Revista de Administração Contemporânea, vol.15, n.4, p. 580-607, jul./ago. 2011.

MATTAR, F. N. Pesquisa de marketing. São Paulo: Atlas, 1996.

MEISTER, J. C. Educação Corporativa. Trad. Maria Claudia Santos Ribeiro Ratto. São Paulo: Pearson Makron Books, 1999.

MORIN, L.; RENAUD, S. Participation in corporate university training: its effect on individual job performance. Canadian Journal of Administrative Sciences, v. 21, n.4, p. 295-306, 2004.

MORITZ, G. O. Planejando por Cenários Prospectivos: a construção de um referencial metodológico em um estudo de casos. 2004. Tese (Doutorado em Engenharia de Produção) - Programa de Pós-Graduação, UFSC, Florianópolis, 2004.

OLIVEIRA, O. V.; FORTE, S. H. A. C. Cenários Prospectivos da Indústria Bancária Brasileira: identificação dos recursos competitivos de utilização mais provável. In: ENCONTRO DE ESTUDOS ESTRATÉGICOS, 4., 2009, Recife. Anais... Recife: ANPAD, 2009.

OLIVEIRA, O. V.; FORTE, S. H. A. C. Identificação dos recursos competitivos de utilização mais provável pela indústria bancária brasileira de 2008 a 2012. Revista de Administração, São Paulo, v. 46, n. 3, p. 243-257, jul./ago./set. 2011. 
PARKER, Phill. The Future ofthe Corporate University. Disponível em $<$ https://knowledge.insead.edu/leadership-organisations/thefuture-of-thecorporate-university-5121\#GsVVBZdvy0IW6bfq.99>. Acesso em 23.07.2017.

PATON, R.; TAYLOR S.; STOREY, J. Corporate Universities and Leadership Development In: STOREY, J. (ed.). Leadership in Organisations: current issues and key trends. London: Routledge, 2004.

PNUD. Programa das Nações Unidas Para o Desenvolvimento. Objetivos de desenvolvimento para o milênio. Disponível em: <http://www.pnud.org.br/odm>. Acesso em: 20 out. 2007.

RANGEL, E. P. Desenvolvimento de uma análise sistêmica de cenários prospectivos para o setor de pelotas de minério de ferro brasileiro. 2012. Dissertação (Mestrado em Engenharia Metalúrgica) - Escola de Engenharia, Universidade Federal de Minas Gerais (UFMG), Belo Horizonte, 2012.

RICHARDSON, R. J. Pesquisa social: métodos e técnicas. São Paulo: Editora Saraiva, 1999.

RODRIGUEZ, M. V. Existe diferença entre universidade corporativa e centro de treinamento? Disponível em: <www.rh.com.br>. Acesso em: 10 out. 2016.

SILVA, A. T. B. et al. A Elaboração de Cenários na Gestão Estratégica das Organizações: um Estudo Bibliográfico. In: SEMINÁRIO EM ADMINISTRAÇÃO, 13. Anais..., 2010. Disponível em: <http://consultoriaprofuturo.com/wpcontent/uploads/2010/09/Elaboracao-decenarios2.pdf >. Acesso em: 5 out. 2016.

SILVA, W. R.; ALFREDO NETO. J.; SANTOS, L. A. Cenários Prospectivos como Ferramenta de Gestão no ensino Superior. $8^{\circ}$ Congresso IFBAE. Anais...Gramado/RS - 18 e 19 de maio de 2015.

SILVERMAN, D. Interpretação de dados qualitativos: métodos para análise de entrevistas, textos e interações. Porto Alegre: Artmed/Bookman, 2009.

THOMAS, J. R.; NELSON, J. K.; SILVERMAN, S. J. Métodos de pesquisa em atividade física. 5. ed. Porto Alegre: Artmed, 2007.

WACK, P. Scenarios: uncharted waters ahead. Harvard Business Review, p. 72-89, Sept./Oct, 1985.

WRIGHT, P.; KROLL, M. J.; PARNELL, J. Administração estratégica: conceitos. São Paulo: Atlas, 2000. 